\title{
Recurrence of Strokes and Associated Factors at Laquintinie Hospital in Douala
}

\author{
Paul-Cedric Mbonda ${ }^{1,}$, , Daniele Mafo ${ }^{2}$, Jacques Doumbe ${ }^{3}$, Callixte Kuate ${ }^{1}$ \\ ${ }^{1}$ Faculty of Medicine and Biomedical Sciences, University of Yaoundé 1, Yaoundé, Cameroon \\ ${ }^{2}$ Faculty of Health Sciences, Mountain University, Banganté, Cameroon \\ ${ }^{3}$ Faculty of Medicine and Pharmaceutical Sciences, University of Douala, Douala, Cameroon
}

Email address:

mbondapaul@gmail.com (Paul-Cedric M.)

${ }^{*}$ Corresponding author

\section{To cite this article:}

Paul-Cedric Mbonda, Daniele Mafo, Jacques Doumbe, Callixte Kuate. Recurrence of Strokes and Associated Factors at Laquintinie Hospital in Douala. Clinical Neurology and Neuroscience. Vol. 5, No. 3, 2021, pp. 46-49. doi: 10.11648/j.cnn.20210503.12

Received: May 22, 2021; Accepted: June 17, 2021; Published: June 25, 2021

\begin{abstract}
OVERVIEW: Stroke is the second leading cause of death in the world and the leading cause of non-traumatic disability in adults. Although the incidence of stroke has steadily declined in developed countries, the incidence in low- and middle-income countries like Cameroon continues to grow, accounting for $85 \%$ of the global burden of stroke. Whenever a stroke occurs, the patient, the patient's family and the physician want to know the risk of recurrence, its severity and the possibility of prevention. Few studies have focused on stroke recurrences and factors associated with Cameroon. OBJECTIVE: To determine the prevalence of recurrent stroke and the factors associated with Laquintinie Hospital in Douala. METHODOLOGY: We conducted a two-year retrospective study from January 1, 2016 to December 31, 2017 and five-month cross-sectional prospective from January 1, 2018 to May 31, 2018 at Laquintinie Hospital in Douala. Included were all patients hospitalized for stroke. RESULTS: We recruited 528 stroke patients, including 75 recidivists with a recurrence prevalence of $14.20 \%$. The average age of recidivism was $65.82 \pm 12.75$ years and $70.6 \%$ female. Factors statistically associated with recurrence were an antecedent of hypertension $(\mathrm{OR}=038$ [0.152-0.98], $\mathrm{P}=0.045)$, the ischemic type of first stroke $(\mathrm{OR}=2.32$ [1.04-5.17] $\mathrm{P}=0.04)$; females $(\mathrm{OR}=2.052$ [1.20-3.4921]; $\mathrm{P}=0.007)$ and poor treatment compliance after first stroke $(\mathrm{OR}=0.399$ [0.160-0.99] $\mathrm{P}=0.042)$. CONCLUSION: About 1 out of $6(14.2 \%)$ survivors of a first stroke have recurrent stroke over the next 2 years at Laquintinie Hospital in Douala. The predictive factors for recurrence in this study were a history of hypertension, the ischemic type of stroke, female gender, and poor adherence to secondary prevention measures.
\end{abstract}

Keywords: Recurrence, Stroke, Risk Factors

\section{Background}

Stroke is one of the main public health problems in the world today, since 2005 it is the second leading cause of death in the world and in sub-Saharan Africa [1]. In Cameroon the one-month stroke mortality rate was $26.7 \%$ in a referral hospital in Douala [2].

Despite the fact that treatment methods have evolved further, we still face recurrences of stroke. Recurrent stroke is defined as the appearance of a new focal neurological deficit with no apparent cause other than that of vascular origin occurring any time after the first stroke [3] or the sudden onset of an exacerbation of a previous focal neurologic deficit with no other apparent cause than vascular origin $>21$ days after the initial stroke.

The risk factors for recurrent stroke are many and diverse. In Singapore in 2013; Sun et al report a prevalence of $15.7 \%$ of recurrence of stroke in five years with age as the main predictor of recurrence [4]. Lekoubou et al found a stroke recurrence prevalence of $14.5 \%$ at the Yaoundé central hospital in Cameroon in 2017 [5].

To our knowledge, few studies have been carried out in Cameroon and even in Africa on this subject. The aim of this study was to determine the prevalence and to research the factors associated with recurrence of strokes at Laquintinie hospital in Douala. 


\section{Methods}

This was a retrospective, cross-sectional descriptive study that took place in the neurology department of Laquintinie Hospital in Douala.

Laquintinie Hospital in Douala, created in 1931, is a 2nd category referral hospital. It is one of the three referral hospitals in the city of Douala, the economic capital of Cameroon with more than three million inhabitants. Due to the relatively low cost of care provided in this health facility, it is very accessible.

The cross-sectional part of the study was carried out over a period of 05 months and the retrospective concerned the use of records of patients hospitalized for stroke over 2 years. Were included in this study, patients who had a recurrent stroke at Laquintinie Hospital in Douala during the study period.

In total, we collected data from 528 patients and identified those who had a recurrence. Sociodemographic information (age, sex, profession, marital status, level of education), information on primary stroke (age of onset, type of stroke, territory concerned, vascular risk factors, treatment administered as well as observance) and information on the recurrence (type of stroke, site, time to recurrence) was taken.

The data were recorded and then analyzed using SPSS software version 20.0. The significance level was set at 0.05 . The chi-square test or Student's $\mathrm{T}$ test used for comparison between different groups and to look for predictors of recurrence.

Ethics Approval

The study was approved by the Regional Ethics Committee and was conducted in accordance with the 1964 Helsinki Declaration and its subsequent amendments.
On 528 patients with stroke recruited in our study, we found 74 cases of recurrence, ie a prevalence of stroke recurrence of $14 \%$. In our study population, the mean age of onset of $1 \mathrm{st}$ stroke was $62.49 \pm 13.36$ years. Regarding recurrence, $75 \%$ of patients were between 55-85 years old, the female sex (70\%) was predominant, and $67.5 \%$ were retired.

The most common stroke risk factors found in patients with recurrent stroke were $90.7 \%$ hypertension, age $>60$ years $(61.3 \%)$, physical inactivity $(61.3 \%)$ menopause $(58.7 \%)$ and obesity (52\%).

Regarding the inaugural stroke we had $70 \%$ ischemia, and during the recurrence $81 \%$ of strokes were ischemic. The vast majority of patients had damage to the middle cerebral artery. Regarding recurrences with hemorrhage, the predominant location was temporo-parietal (74\%).

Lacunar infarcts (64.7\%), atherosclerosis (23.5\%) and atrial fibrillation were the most common etiologies among ischemic stroke recurrences. High blood pressure was the most common etiology in recurrent hemorrhagic stroke.

As regards the treatment, of all the patients with a recurrent stroke, $65 \%$ were not complying with the treatment prescribed after the 1 st stroke.

The time between the first stroke and recurrence was 1 to 2 years in $37.8 \%$ of patients, more than 3 years in $28.4 \%$ of patients, and $14.9 \%$ of patients had recurrence within six months of the first stroke.

The follow-up of our patients who had a recurrence of stroke was marked by the occurrence of $23 \%$ of deaths.

After logistic regression analysis (Table 1), the factors statistically associated with stroke recurrence were: female sex $(\mathrm{P}=0.02)$, ischemic stroke $(\mathrm{P}=0.007)$, history of high blood pressure $(\mathrm{P}=0.001)$, and non-compliance with treatment after the 1 st stroke $(\mathrm{P}=0.042)$.

\section{Results}

Table 1. Univariate analysis looking for factors associated with stroke recurrence.

\begin{tabular}{|c|c|c|c|c|}
\hline Characteristics & Recurrent stroke Frequency (\%) & New stroke Frequency (\%) & P-value & OR [IC 95\%] \\
\hline Female & $52(70,3)$ & $243(53 \%)$ & 0,007 & $2,052[1,2062-3,4921]$ \\
\hline Ischemic Stroke & $61(82,4)$ & $349(66,1)$ & 0,007 & $0,410[0,22-0,7]$ \\
\hline Past history of high blood pressure & $59(90,5)$ & $270(62,3)$ & 0,001 & $2,680[1,4756-4,8692]$ \\
\hline Past history of diabetes & $7(13,5)$ & $44(9,7)$ & 0,950 & $0,973[0,421-2,251]$ \\
\hline Treatment compliance & $40(64,86)$ & $149(35,23)$ & 0,042 & $0,399[0,160-0,992]$ \\
\hline Taking antihypertensive drugs & $44(93,2)$ & $200(46,2)$ & 0,014 & $1,862[1,130-3,0701]$ \\
\hline
\end{tabular}

\section{Discussion}

The objective of our study was to determine the prevalence of recurrence of stroke and the associated factors at Laquintinie Hospital, in order to contribute to the fight against this group of disease.

The mean age of stroke was $62.49+/-13.36$ years at the first episode and $65.82 \pm 12.75$ years at recurrence. The youngest patient was 38 years old and oldest 87 years old, this average is almost similar to that obtained by Oh et al in 2017 in a South Korean study on stroke recurrence where the average age was $63.9 \pm 13$ years [6]. This shows that the trends in the age of stroke recurrence are quite similar around the world. In similar studies carried out in Ireland [7], Australia [8] the average age was higher but these observed differences could be explained by a greater aging of the population of developed countries compared to the general population of Cameroon.

Of our patients who had a recurrence of stroke, the female sex was the most represented with $70.3 \%$ of cases. However, the majority of studies on recurrence of stroke report a predominance of the male sex, the small size of our sample could explain the observed difference.

Regarding the type of stroke, ischemia was found most often in the first episode $(66.1 \%)$ and recurrence $(81.3 \%)$. This is in 
line with the trends described in the literature by Kuate et al in 2016, which mainly found the ischemic type at 53\% [9].

The cardiovascular risk factors most found in our study during the first episode as well as after recurrence are high blood pressure, sedentary lifestyle, age over 60 years, obesity, male sex, menopause, alcoholism and smoking. Modrego et al in 2000 in Spain found as vascular risk factors: high blood pressure, diabetes, alcoholism and chronic smoking, heart diseases such as atrial fibrillation, cardiomyopathies, coronary artery disease [10]. And according to Liu et al, the presence of cardiovascular risk factors increases the risk of stroke recurrence [11].

Lacunar infarctions were the predominant etiology of ischemic strokes and their recurrences in our population followed by atherosclerosis, apart from the order of frequency, these same aetiologies are cited by Modrego who found atherosclerosis as the primary cause of recurrence, then cardioembolic causes and finally lacunar lesions [10].

Among the patients who had a first vascular event, only $28 \%$ were taking their treatment correctly before the recurrence. This low percentage of compliance is probably due in part to financial difficulties, but also a post-stroke depressive syndrome could be involved, indeed, according to Ezema et al, depression is found in $30-50 \%$ of patients after a stroke and would increase the risk of recurrence [12]

The mortality rate after the first episode of stroke was $15 \%$, and after recurrence this rate rises to $23 \%$, which shows that mortality is more important after the recurrence. These results are similar to those obtained at the Yaoundé Central Hospital by Lekoubou et al, who reported a first stroke mortality rate of $19.6 \%$ and recurrence of $20.6 \%$ [5].

Montanaro et al in 2018 in Brazil reported a death rate among recurrent stroke of $10 \%$ in a sample smaller than ours and prospective follow-up over at least 3 years [13]. Callaly et al [7] in a cohort study reported a mortality rate of $38.5 \%$ over two years. Death rates vary from study to study but are higher in developing countries due to scarce and expensive means of exploration, the lack of a health insurance system and even poor adherence to preventative measures.

The majority of patients relapsed within one to two years of the first episode. Most studies of stroke recurrence have found a higher rate of recurrence within 2 years [7].

Our study shows that the prevalence of stroke recurrence at Laquintinie Hospital in Douala over a period of 2 years and 5 months is $14 \%$. This figure is close to that reported by Pan et al in 2016 in China with $13 \%$ of recurrence in one year [14], as well as that found by Zhao et al in 2015 which was $12.9 \%$ [15], $15 \%$ of recurrence of stroke in the study by Sun et al in Singapore in Thailand in 2015 [4]. Lekoubou et al in 2017 in Yaoundé, Cameroon, found a stroke recurrence rate of $14.5 \%$ over a period of 13 years (1999-2012). Similar recurrence rates are reported by Hankey et al in Australia who found $8.8 \%$ recurrence over 6 months, $13.4 \%$ over two years [8]. On the other hand, lower recurrence rates after stroke are reported by Hillen et al in England with $5.58 \%$ over 12 months [16]. Our study, being cross-sectional and hospital-based, only took into account patients readmitted to our hospital, and could exclude patients followed in other hospitals in the city or in the country, or even those who are lost to follow-up.

The factors associated with recurrence in multivariate analysis are: high blood pressure, non-compliance with secondary prevention treatment, to which are added the female sex and the ischemic type in univariate analysis.

High blood pressure is recognized in the literature as a cardiovascular risk factor and the leading risk factor for stroke [17], this pathology is also incriminated in several studies as a factor associated with recurrence of stroke in China [18], in Europe [19] in Africa (Nigeria) [20], the frequent involvement of this factor in recurrent strokes may be explained by poor adherence to antihypertensive drugs.

\section{Conclusion}

We can conclude at the end of our study that stroke recurrences are frequent at Laquintinie Hospital in Douala, affecting nearly $14 \%$ of patients after a first stroke. These recurrences are more frequent in patients suffering from ischemic stroke, with uncontrolled high blood pressure, and who are not complying with secondary prevention measures. This high recurrence rate reminds us of the need for secondary prevention measures to be introduced as early as possible and of the importance for practitioners to communicate well with patients and follow them rigorously.

\section{Acknowledgements}

We are grateful to the staff at Laquintinie Hospital who helped us in this work. Authors have no financial, political, personal, religious, ideological, academic, intellectual, commercial or any other conflicts of interest to declare in relation to this manuscript.

\section{References}

[1] Owolabi M, Sarfo FS, Akinyemi R, Gebreyohanns M, Ovbiagele B. TheSub-Saharan Africa Conference on Stroke (SSACS): Anidea whose time has come. JNeurolSci. 2019; 400: 194-198.

[2] Kuate C, Kenmogne M, Doumbe J, Mapoure Y, Tchaleu B, Noubissi G, et al. Stroke mortality and its determinants in a referral hospital at Douala (Cameroon). Health Sc Dis. 2016; 17 (1): 585 .

[3] Frans Kauw, Richard A P Takx, Hugo W A M de Jong, Birgitta K Velthuis, L Jaap Kappelle, Jan W Dankbaar. Clinical and Imaging Predictors of Recurrent Ischemic Stroke: A Systematic Review and Meta-Analysis Cerebrovasc Dis. 2018; 45 (5-6): 279-287.

[4] Sun Y, Lee SH, Heng BH, Chin VS. 5-year survival and rehospitalization due to stroke recurrence among patients with hemorrhagic or ischemic strokes in Singapore. BMC Neurol. 2013; 13: 133. 
[5] Lekoubou A, Nkoke C, Dzudie A, Kengne AP. Recurrent Stroke and Early Mortality in an Urban Medical Unit in Cameroon. J Stroke Cerebrovasc. Dis off J Natl Stroke Assoc. 2017; 26 (8): 1689-94.

[6] Oh J-S, Bae H-G, Oh HG, Yoon S-M, Doh J-W, Lee K-S. The Changing Trends in Age of First-Ever or Recurrent Stroke in A Rapidly Developing Urban Area during 19 Years. J Neurol Neurosci. 2017; 8 (4): 206.

[7] Callaly E, Ni Chroinin D, Hannon N, Marnane M, Akijian L, Sheehan O, et al. Rates, Predictors, and Outcomes of Early and Late Recurrence After Stroke: The North Dublin Population Stroke Study. Stroke. 2016; 47 (1): 244-6.

[8] Hankey GJ, Jamrozik K, Broadhurst RJ, Forbes S, Burvill PW, Anderson CS, et al. Long-Term Risk of First Recurrent Stroke in the Perth Community Stroke Study. Stroke. 1998; 29 (12): 2491-500.

[9] Kuate C, Mapoure Y, Lauriane G-M, Jacques D, Gustave N-D, Ii J, et al. Stroke mortality and its determinants in a tertiary care Hospital at Douala (Cameroon). Health Sci Dis. 2016; 17: 16.

[10] Modrego PJ, Pina MA, Fraj MM, Llorens N. Type, causes, and prognosis of stroke recurrence in the province of Teruel, Spain. A 5-year analysis. Clin Neurophysiol. 2000; 21 (6): 355-60.

[11] Lin B, Zhang Z, Mei Y, Wang C, Xu H, Liu L, Wang W. Cumulative risk of stroke recurrence over the last 10 years: a systematic review and meta-analysis. Neurol Sci. 2021; 42(1): $61-71$.

[12] Ezema CI, Akusoba PC, Nweke MC, Uchewoke CU, Agono J, Usoro G. Influence of Post Stroke Depression on Functional Independence in Activities of Daily Living. Ethiop J Health Sci. 2019; 29(1): 841-846.
[13] Montanaro VVA, Hora TF, da Silva CM, Santos CV, Lima MIR, Negrao EM, et al. Mortality and Stroke Recurrence in a Rehabilitation Cohort of Patients with Cerebral Infarcts and Chagas Disease. Eur Neurol. 2018; 79: 177-184.

[14] Pan Y, Wang Y, Li H, Gaisano HY, Wang Y, He Y. Association of Diabetes and Prognosis of Minor Stroke and Its Subtypes: A Prospective Observational Study. PloS One. 2016; 11 (4): e0153178.

[15] Zhao L, Wang R, Song B, Tan S, Gao Y, Fang H, et al. Association between atherogenic dyslipidemia and recurrent stroke risk in patients with different subtypes of ischemic stroke. Int J Stroke. 2015; 10 (5): 752-8.

[16] Hillen T, Coshall C, Tilling K, Rudd AG, McGovern R, Wolfe CDA. Cause of Stroke Recurrence Is Multifactorial: Patterns, Risk Factors, and Outcomes of Stroke Recurrence in the South London Stroke Register. Stroke. 2003; 34 (6): 1457-63.

[17] Boan AD, Lackland DT, Ovbiagele B. Lowering of blood pressure for recurrent stroke prevention. Stroke. 2014; 45 (8): 2506-13.

[18] Fu G-R, Yuan W-Q, Du W-L, Yang Z-H, Fu N, Zheng H-G, et al. Risk Factors Associated with Recurrent Strokes in Young and Elderly Patients: A Hospital-based Study. Int J Gerontol. 2015; 9 (2): 63-6.

[19] Jona T Stahmeyer, Sarah Stubenrauch Siegfried Geyer, Karin Weissenborn, Sveja Eberhard. The Frequency and Timing of Recurrent Stroke: An Analysis of Routine Health Insurance Data. Dtsch Arztebl Int. 2019Oct18; 116 (42): 711-717.

[20] Osemwegie N, Danesi M. Predictors of Stroke Recurrence at Lagos University Teaching Hospital, Nigeria. Neurology. 2018; 90 (15): 2-222. 